\title{
WEAK AMENABILITY OF CAT(0)-CUBICAL GROUPS
}

\author{
ERIK GUENTNER AND NIGEL HIGSON
}

\begin{abstract}
We prove that if $\mathrm{G}$ is a discrete group that admits a metrically proper action on a finitedimensional CAT( 0 ) cube complex $X$, then $G$ is weakly amenable. We do this by constructing uniformly bounded Hilbert space representations $\pi_{z}$ for which the quantities $z^{\ell(g)}$ are matrix coefficients. Here $\ell$ is a length function on $G$ obtained from the combinatorial distance function on the complex X.
\end{abstract}

\section{INTRODUCTION}

Let $\mathrm{G}$ be a countable discrete group and denote by $C_{\lambda}^{*}(\mathrm{G})$ the reduced $\mathrm{C}^{*}$-algebra of $\mathrm{G}$. Every finitely supported function $\phi: G \rightarrow \mathbb{C}$ defines a bounded linear operator

$$
M_{\phi}: C_{\lambda}^{*}(G) \rightarrow C_{\lambda}^{*}(G)
$$

by the formula $\left(M_{\phi} f\right)(g)=\phi(g) f(g)$, as well as bounded linear operators

$$
M_{\phi}^{(n)}: M_{n}\left(C_{\lambda}^{*}(G)\right) \rightarrow M_{n}\left(C_{\lambda}^{*}(G)\right)
$$

between matrix algebras by applying $M_{\phi}$ to each matrix entry. The completely bounded multiplier norm of $\phi$ is defined to be the quantity

$$
\|\phi\|_{\mathrm{cb}}=\sup _{\mathfrak{n}}\left\|M_{\phi}^{(\mathfrak{n})}\right\| .
$$

See [CH89, Pis95]. A countable discrete group is weakly amenable [CH89] if there exists a sequence of finitely supported functions on $\mathrm{G}$ converging pointwise to 1 and consisting of functions which are uniformly bounded in the completely bounded multiplier norm. 1 Recall that a countable discrete group $\mathrm{G}$ is amenable if and only if there exists a sequence of finitely supported, positivedefinite functions on $\mathrm{G}$ converging pointwise to the constant function 1 on $\mathrm{G}$. It may be shown that the completely bounded multiplier norm of a positive-definite function $\phi$ is equal to $\phi(e)$. As a result, every weakly amenable group is amenable.

The notion of amenability may be broadened in a different way. A $c_{0}$-function on a discrete set is a function that extends to a continuous function on the one-point compactification with value 0 at infinity. A countable discrete group is said to have the Haagerup property $\left[\mathrm{CCJ}^{+} 01\right]$ if there exists a sequence of positive-definite $c_{0}$-functions on $G$ converging pointwise to the constant function 1 on G. Since every finitely supported function is a $c_{0}$-function, every amenable group has the Haagerup property.

Date: June 30, 2021.

The authors were partially supported by grants from the U.S. National Science Foundation.

${ }^{1}$ This is not the standard definition, but see [Haa86, HK94]. 
Michael Cowling conjectures that every countable discrete group with the Haagerup property is weakly amenable $\left[\mathrm{CCJ}^{+} 01\right]$. He further conjectures that the Cowling-Haagerup constant of any such group is 1 , which means that there exists a sequence of finitely supported functions on $G$ converging pointwise to 1 and consisting of functions whose completely bounded multiplier norm is bounded by 1. The conjecture is supported by the cases of Coxeter groups [Jan02] and discrete subgroups of $\mathrm{SO}(n, 1)$ [dCH85] and $\mathrm{SU}(n, 1)$ [Cow83].

In this paper we shall consider groups which admit an action on a finite-dimensional CAT(0)cubical complex which is proper in the sense that for every vertex $x$ in the complex, the function $\mathrm{d}(\mathrm{gx}, \mathrm{x})$ on $\mathrm{G}$ is proper. We shall refer to these groups as CAT(0)-cubical groups. Niblo and Reeves [NR97, NR98b] proved that CAT(0)-cubical groups have the Haagerup property. We shall prove the following result:

Theorem. Every CAT(0)-cubical group is weakly amenable, with Cowling-Haagerup constant one.

Our theorem adds evidence in favor of Cowling's conjecture. In fact it might be argued that the class of CAT(0)-cubical groups is quite close to the class of all groups with the Haagerup property. It is known that a discrete group has the Haagerup property if and only if it acts properly on a measured space with walls (see [CMV04] for the precise assertion). Furthermore, a discrete group acts on a combinatorial space with walls if and only if it acts on a CAT(0) cube complex, although possibly an infinite-dimensional CAT(0) cube complex. See [CN05, Nic04]. Thus, disregarding the issue of infinite-dimensionality, the difference between the class of groups with the Haagerup property and the class of CAT(0)-cubical groups comes down to the difference between combinatorial and measured spaces with walls. As Graham Niblo has explained to us, the distinction is analogous to that between trees and $\mathbb{R}$-trees.

Unfortunately the possible infinite-dimensionality of CAT $(0)$ cube complexes can pose serious problems. For example Farley [Far03] proved that Thompson's group F has the Haagerup property by exhibiting a metrically proper action of F on a certain CAT(0) cube complex. However Farley's complex, although locally finite-dimensional, is infinite-dimensional and our theorem above does not apply. The question of whether or not $F$ is weakly amenable remains open.

In order to prove the weak amenability of CAT(0)-cubical groups we shall rely on a result of Valette [Val93] that makes it possible to deduce weak amenability from the existence of a suitable holomorphic family of uniformly bounded representations. Denote by $\mathbb{D}$ the open unit disk in the complex plane. A family $\left\{\pi_{z}\right\}_{z \in \mathbb{D}}$ of representations of a discrete group $G$ into the bounded invertible operators on a Hilbert space $\mathcal{H}$ is holomorphic if for every element $\mathrm{g} \in \mathrm{G}$ and every pair of vectors $\xi, \xi^{\prime} \in \mathcal{H}$ the matrix coefficient

$$
\phi(z)=\left\langle\pi_{z}(g) \xi, \xi^{\prime}\right\rangle
$$

is a holomorphic function on $\mathbb{D}$. This definition is explored in [ $\mathrm{dCH} 85$ ].

Theorem (See [Val93]). Let $\mathrm{G}$ be a countable discrete group. Assume that there exists a proper function $\ell: \Gamma \rightarrow \mathbb{N}$ with $\ell(e)=0$ and a holomorphic family $\left\{\pi_{z}\right\}_{z \in \mathbb{D}}$ of uniformly bounded representations of $\mathrm{G}$ on a Hilbert space $\mathcal{H}$ such that $\pi_{\mathrm{t}}$ is unitary for $0<\mathrm{t}<1$. If there exists a 
vector $\xi \in \mathcal{H}$ such that $z^{\ell(g)}=\left\langle\pi_{z}(\mathrm{~g}) \xi, \xi\right\rangle$ for every $z \in \mathbb{D}$ and every $\mathrm{g} \in \mathrm{G}$, then $\mathrm{G}$ is weakly amenable with Cowling-Haagerup constant one.

We shall construct the required representations following a method of Pimsner [Pim87] and Valette [Val90a] that we shall now outline. Let $X$ be a set equipped with an action of $G$ and let $\pi$ be the natural permutation representation of $G$ on $\ell^{2}(X)$. A function

$$
c: X \times X \rightarrow \mathcal{B}\left(\ell^{2}(X)\right)
$$

is a cocycle for $\pi$ if

(a) $c(x, x)=1$

(b) $c(v, x) c(x, y)=c(v, y)$

(c) $\pi(g) c(x, y) \pi(g)^{-1}=c(g \cdot x, g \cdot y)$

for all $\nu, x, y \in X$ and all $g \in G$. If $c$ is a cocycle for $\pi$, and if $x \in X$, then the formula

$$
\pi_{\mathrm{c}}(\mathrm{g})=\mathrm{c}(\mathrm{x}, \mathrm{g} x) \pi(\mathrm{g})
$$

defines a representation of $G$ into the bounded invertible operators on $\ell^{2}(X)$. If the cocycle $c$ is uniformly bounded, meaning that

$$
\sup _{x, y \in X}\|c(x, y)\|<\infty,
$$

then $\pi_{c}$ is uniformly bounded. If the cocycle is unitary, meaning that $c(x, y)=c(y, x)^{*}$ for all $x$ and $y$, then the representation $\pi_{c}$ is unitary.

We shall take $X$ to be the set of vertices of a finite-dimensional CAT $(0)$ cube complex on which $\mathrm{G}$ acts, and we shall construct a family of cocycles $\left\{c_{z}\right\}_{z \in \mathbb{D}}$ using the geometry of CAT( 0$)$ cube complexes. In doing so we shall closely follow Pimsner and Valette, who constructed uniformly bounded representations in this way when $X$ is the set of vertices of a tree [Pim87, Val90a], and Januszkiewicz, who extended their results to products of trees [Jan93]. The same cocycle has also been analyzed very carefully by Brodzki, Niblo and Valette in unpublished work. The construction of the cocycle is straightforward; the proof that the cocycle is uniformly bounded is more difficult and this is our contribution.

\section{Overview of THE PROOF}

To prepare the reader for the more complicated case of CAT $(0)$ cube complexes, we shall rapidly review the construction of Pimsner and Valette for trees (which are simple examples of CAT(0) cube complexes). Let $X$ be the set of vertices of a simplicial tree on which a group $G$ acts. If $z \in \mathbb{D}$, then define $w \in \mathbb{C}$ by the equation $z^{2}+w^{2}=1$, or $w=\sqrt{1-z^{2}}$, where we use the branch of the square root function which is holomorphic on the complement of the negative real axis and which is positive on the positive real axis. If $x, y \in X$ are the vertices of an edge, then define $c_{z}(x, y) \in \mathcal{B}\left(\ell^{2}(X)\right)$ by

$$
c_{z}(x, y) \delta_{v}= \begin{cases}w \delta_{x}-z \delta_{y}, & v=x \\ w \delta_{y}+z \delta_{x}, & v=y \\ \delta_{v}, & \text { otherwise }\end{cases}
$$


where $\delta_{v}$ denotes the Dirac function at the vertex $v$. In other words, $c_{z}(x, y)$ is given by the matrix

$$
\left(\begin{array}{cc}
w & z \\
-z & w
\end{array}\right)
$$

on the two-dimensional subspace spanned by the ordered basis $\left(\delta_{x}, \delta_{y}\right)$ and it is the identity on the orthogonal complement of this subspace. Notice that

$$
c_{z}(x, y)=c_{z}(y, x)^{-1}
$$

while if $z$ is real, then $c_{z}(x, y)=c_{z}(y, x)^{*}$, and hence $c_{z}(x, y)$ is unitary. If $x$ and $y$ are arbitrary vertices in the tree, then define $c_{z}(x, y) \in \mathcal{B}\left(\ell^{2}(X)\right)$ by

$$
c_{z}(x, y)=c_{z}\left(v_{0}, v_{1}\right) c_{z}\left(v_{1}, v_{2}\right) \cdots c_{z}\left(v_{n-1}, v_{n}\right) \text {, }
$$

where $x=v_{0}, v_{1}, \ldots, v_{n}=y$ are the vertices along any edge-path from $x$ to $y$. It follows from (1.1) and the basic geometry of trees that this definition is independent of the path chosen between $x$ and $y$, and it follows from this that $c_{z}$ is a cocycle for $\pi$.

Fixing a vertex $x \in X$ we obtain representations $\pi_{z}(g)=c_{z}(x, g x) \pi_{g}$. This is a holomorphic family - indeed for each $g$, the operator $\pi_{z}(g)$ is a polynomial in $z$ and $w$ with coefficients in $\mathcal{B}\left(\ell^{2}(X)\right)$. Moreover, if we define $\ell(g)$ to be $d(x, g x)$, the edge-path length between $x$ and $g x$, then it is easy to check that $z^{\ell(g)}=\left\langle\pi_{z}(g) \delta_{x}, \delta_{x}\right\rangle$ (see for example the computations following Lemma 1.1 below). If $z$ is real, then $\pi_{z}$ is a unitary representation.

In order to apply Valette's theorem it remains to show that for general $z \in \mathbb{D}$, the representation $\pi_{z}$ is uniformly bounded. We shall briefly indicate a series of lemmas sufficient to prove this that are representative of what we are able to accomplish for general CAT $(0)$ cube complexes. Denote by $c_{a b}$ the matrix entries of $c_{z}(x, y)$, so that if $b \in X$, then

$$
c_{z}(x, y) \delta_{b}=\sum_{a \in X} c_{a b} \delta_{a},
$$

or equivalently $c_{a b}=\left\langle c_{z}(x, y) \delta_{b}, \delta_{a}\right\rangle$.

1.1. Lemma. Each nonzero matrix entry $\mathrm{c}_{\mathrm{ab}}$ has the form $\mathrm{c}_{\mathrm{ab}}= \pm z^{\mathrm{k}} \mathrm{w}^{\ell}$ where $\mathrm{k}=\mathrm{d}(\mathrm{a}, \mathrm{b})$ and where $\ell \leq 2$.

This is the geometric heart of the uniform boundedness argument. For trees it may be proved by explicitly computing all the coefficients $c_{a b}$. Let $v_{0}, \ldots v_{n}$ be the vertices on the geodesic path from $x$ to $y$. If $b$ does not belong to this path, then it is easy to see that $c_{z}(x, y) \delta_{b}=\delta_{b}$. If $b=v_{n}$, then

$$
\begin{aligned}
c_{z}(x, y) \delta_{b} & =c_{z}\left(v_{0}, v_{1}\right) \cdots c_{z}\left(v_{n-1}, v_{n}\right) \delta_{v_{n}} \\
& =c_{z}\left(v_{0}, v_{1}\right) \cdots c_{z}\left(v_{n-2}, v_{n-1}\right)\left(w \delta_{v_{n}}+z \delta_{v_{n-1}}\right) \\
& =c_{z}\left(v_{0}, v_{1}\right) \cdots c_{z}\left(v_{n-3}, v_{n-2}\right)\left(w \delta_{v_{n}}+w z \delta_{v_{n-1}}+z^{2} \delta_{v_{n-2}}\right) \\
& \vdots \\
& =w \delta_{v_{n}}+w z \delta_{v_{n-1}}+w z^{2} \delta_{v_{n-2}}+\cdots+w z^{n-1} \delta_{v_{1}}+z^{n} \delta_{v_{0}}
\end{aligned}
$$


If $\mathrm{b}=v_{\mathrm{i}}$ with $\mathrm{i}<\mathrm{n}$, then $\mathrm{c}_{z}\left(v_{\mathrm{i}+1}, v_{\mathrm{i}+2}\right) \cdots \mathrm{c}_{z}\left(v_{\mathrm{n}-1}, v_{\mathrm{n}}\right) \delta_{\mathrm{b}}=\delta_{\mathrm{b}}$ and so

$$
\begin{aligned}
c_{z}(x, y) \delta_{b} & =c_{z}\left(v_{0}, v_{1}\right) \cdots c_{z}\left(v_{i}, v_{i+1}\right) \delta_{v_{i}} \\
& =c_{z}\left(v_{0}, v_{1}\right) \cdots c_{z}\left(v_{i-1}, v_{i}\right)\left(-z \delta_{v_{i+1}}+w \delta_{v_{i}}\right) \\
& =c_{z}\left(v_{0}, v_{1}\right) \cdots c_{z}\left(v_{i-2}, v_{i-1}\right)\left(-z \delta_{v_{i+1}}+w^{2} \delta_{v_{i}}+z w \delta_{v_{i-1}}\right) \\
& \vdots \\
& =-z \delta_{v_{i+1}}+w^{2} \delta_{v_{i}}+w^{2} z \delta_{v_{i-1}}+\cdots+w^{2} z^{n-i-1} \delta_{v_{1}}+w z^{n-i} \delta_{v_{0}},
\end{aligned}
$$

The lemma follows by inspection of these formulas. In the general case of cube complexes such direct computations are not so easy, and we shall have to work harder to obtain weaker (but adequate) results. But proceeding with the argument for trees, we can now rapidly conclude that the cocycle $c_{z}$ is uniformly bounded.

1.2. Lemma. Let $\mathrm{x}, \mathrm{y} \in \mathrm{X}$, and let $\mathrm{k} \geq 0$. For every $\mathrm{b} \in \mathrm{X}$

$$
\#\left\{a \in X: c_{a b}= \pm z^{k} w^{\ell} \text { for some } \ell \text { and all } z \in \mathbb{D}\right\} \leq 2
$$

and for every $\mathrm{a} \in \mathrm{X}$

$$
\#\left\{\mathrm{~b} \in \mathrm{X}: \mathrm{c}_{\mathrm{ab}}= \pm z^{\mathrm{k}} \mathrm{w}^{\ell} \text { for some } \ell \text { and all } z \in \mathbb{D}\right\} \leq 2 .
$$

Both assertions follow from the fact that for any given vertex $v$ on a geodesic interval $[x, y]$ and any $k$, there are at most two vertices on the interval which are exactly distance $k$ away from $v$.

1.3. Proposition. For every $z \in \mathbb{D}$ and every $x, y \in X$ the quantity $\left\|c_{z}(x, y)\right\|$ is bounded by $4(1-|z|)^{-1}$.

Proof. Let $\mathrm{d}(x, y)=\mathrm{N}$. Decompose the operator $c_{z}(x, y)$ on the $\ell^{2}$-space of the interval $[x, y]$ as a sum

$$
\mathrm{c}_{z}^{(0)}+z \mathrm{c}_{z}^{(1)}+z^{2} \mathrm{c}_{z}^{(2)}+\cdots+z^{\mathrm{N}} \mathrm{c}_{z}^{(\mathrm{N})}
$$

where the operator $c_{z}^{(k)}$, contains only matrix entries of the form $\pm w^{\ell}$ or zero. Since $\ell \leq 2$, each matrix coefficient of $c_{z}^{(k)}$ absolute value 2, or less. Furthermore, by the previous lemma each row and column of $c_{z}^{(k)}$ has at most two non-zero entries. But a matrix which has at most $A$ nonzero entries in each row and column, each of absolute value $B$ or less, determines a operator of norm $A B$ or less. Hence $\left\|c_{z}^{(k)}\right\| \leq 4$, for every $k \geq 0$, so that

$$
\left\|c_{z}(x, y)\right\| \leq 4 \sum_{0}^{N}|z|^{k}
$$

and the proposition follows.

For cube complexes we shall obtain polynomial bounds in place of the absolute bounds in Lemma 1.2, but these will be adequate to carry through a version of the argument in the proof of Proposition 1.3 .

In the case of trees, putting everything together, we obtain the following result (of Szwarc [Szw91] and Valette [Val90b]): 
1.4. Theorem. Suppose that a discrete group $\mathrm{G}$ acts on a tree and that for some vertex $\mathrm{x}$ in the tree the function $\mathrm{g} \mapsto \mathrm{d}(\mathrm{x}, \mathrm{gx})$ is proper on $\mathrm{X}$. Then $\mathrm{G}$ is weakly amenable with Cowling-Haagerup constant 1.

\section{CAT(0) CUBE COMPLEXES}

In this section we shall rapidly review the notion of cube complex and collect the results about them that we shall need.

A cube complex [Gro87, BH99, NR98a] is a set X, called the set of vertices, together with a collection of finite subsets of $X$, called the cubes of $X$, such that: every single-element set is a cube; the intersection of any two cubes is a cube; and for every cube $C$, there is an integer $n \geq 0$ and a bijection from $C$ to the vertices of the standard cube $[0,1]^{n}$ in $\mathbb{R}^{n}$ such that the cubes in $X$ that are subsets of $\mathrm{C}$ correspond precisely to the sets of vertices of the faces (of all dimensions) of the standard cube. The cubes with 2 elements are called edges; those with 4 elements are squares. A cube complex $X$ is finite-dimensional if its cubes are uniformly bounded in cardinality.

Every cube complex has a natural geometric realization in which each cube $C$ is replaced by a unit cube in $\mathbb{R}^{n}$. This geometric realization has a natural metric which restricts to the standard metric on each Euclidean cube. A (globally) non-positively curved cube complex, or CAT $(0)$ cube complex is a cube complex whose geometric realization is a $\mathrm{CAT}(0)$ metric space [BH99]. For a complex whose geometric realization is simply connected the non-positive curvature condition can be phrased combinatorially [Gro87, BH99].

The CAT(0) condition has important consequences which we shall access via the notion of hyperplane. A hyperplane in $\mathrm{X}$ is an equivalence class of edges under the equivalence relation generated by the relation

$$
\{x, y\} \sim\left\{x^{\prime}, y^{\prime}\right\} \quad \Leftrightarrow \quad\left\{x, x^{\prime}, y, y^{\prime}\right\} \text { is a square. }
$$

This definition is a combinatorial proxy for the notion of geometric hyperplane in the geometric realization of $X$ : a geometric hyperplane is the union of an equivalence class of midplanes of cubes under the equivalence relation generated by the relation

$$
M_{1} \sim M_{2} \quad \Leftrightarrow \quad M_{1} \cap M_{2} \text { is the midplane of a cube. }
$$

See [NR98a] for more information and some helpful illustrations. The hyperplane associated to a geometrical hyperplane is the set of all edges bisected by the geometric hyperplane, and every hyperplane arises in this way. The geometric point of view suggests the following terminology: if an edge $\{x, y\}$ belongs to a hyperplane $H$, then we shall say that it crosses $H$; that $H$ separates $x$ from $y$; that $x$ and $y$ are opposite one another across $H$; that $x$ and $y$ are adjacent to $H$; and that $H$ is adjacent to $x$ and $y$.

The most significant consequence of the $\operatorname{CAT}(0)$ condition is that if $X$ is a $\operatorname{CAT}(0)$ cube complex, then every geometric hyperplane separates the geometric resolution of $X$ into precisely two path components [Sag95, Thm 4.10]. This can be expressed combinatorially as follows. A sequence of vertices $v_{0}, \ldots, v_{n}$ in $X$ is an edge-path if each $\left\{v_{i}, v_{i+1}\right\}$ is an edge in $X$. The path crosses a hyperplane $H$ if one if its edges belongs to $H$. Every hyperplane $H$ in a CAT(0) cube 
complex partitions the vertices of $X$ into precisely two sets, such that every edge path from a vertex in one component to a vertex in the other crosses $H$, and such that every pair of vertices in either one of the components is connected by an edge path that does not cross $\mathrm{H}$. These two sets are the half-spaces determined by $\mathrm{H}$ and will they be denoted $\mathrm{H}_{ \pm}$(this involves making an arbitrary choice of which half-space will be $\mathrm{H}_{+}$and which $\mathrm{H}_{-}$; having made such a choice we shall say that $\mathrm{H}$ is oriented). We shall say that the hyperplane $\mathrm{H}$ separates two vertices from one another if one vertex lies in each of the components $H_{ \pm}$. If $x$ and $y$ are vertices of a CAT(0) cube complex $X$, then we shall denote by $\mathfrak{H}(x, y)$ the set of all hyperplanes that separate $x$ from $y$.

The distance $\mathrm{d}(\mathrm{x}, \mathrm{y})$ between two vertices $\mathrm{x}$ and $\mathrm{y}$ of a cube complex $\mathrm{X}$ is the minimum length $n$ of an edge-path $v_{0}, \ldots, v_{n}$ connecting them. An edge-path from $x$ to $y$ is geodesic if it achieves this minimum length.

2.1. Proposition ([Sag95], Thm. 4.13). An edge-path in a CAT(0) cube complex from $x$ to $y$ crosses each hyperplane in $\mathfrak{H}(x, y)$. An edge-path from $x$ to $y$ is geodesic if and only if it crosses only the hyperplanes in $\mathfrak{H}(x, y)$, and crosses each one of those each exactly once.

It follows that $d(x, y)=\# \mathfrak{H}(x, y)$.

2.2. Corollary. Let $\mathrm{x}, \mathrm{y}$ and $v$ be vertices in a $\mathrm{CAT}(0)$ cube complex. The following are equivalent.

(a) $v$ lies on a geodesic from $x$ to $y$.

(b) $\mathrm{d}(x, y)=\mathrm{d}(x, v)+\mathrm{d}(v, y)$.

(c) $\mathfrak{H}(x, v) \cap \mathfrak{H}(v, y)=\emptyset$.

(d) $\mathfrak{H}(x, v) \cap \mathfrak{H}(v, y)=\emptyset$ and $\mathfrak{H}(x, y)=\mathfrak{H}(x, v) \cup \mathfrak{H}(v, y)$

(e) $\mathfrak{H}(x, v) \subseteq \mathfrak{H}(x, y)$

(f) $\mathfrak{H}(x, v) \subseteq \mathfrak{H}(x, y)$ and $\mathfrak{H}(v, y) \subseteq \mathfrak{H}(x, y)$

A corner move transforms an edge-path by changing a string $u, v, w$ in the path into $u, v^{\prime}, w$, where $\left\{u, v, v^{\prime}, w\right\}$ is a square. A corner move does not alter the length of a edge-path or its endpoints.

2.3. Proposition ([Sag95], Thm. 4.6). Any two geodesic edge-paths in a CAT(0) cube complex with the same endpoints differ by a sequence of corner moves.

A simple cancellation in an edge-path replaces a string $v, v^{\prime}, v$ by the singleton string $v$.

2.4. Proposition. Any two paths in a CAT(0) cube complex with the same endpoints are related by a sequence of corner moves and simple cancellations.

To prove this we shall need an additional fact about hyperplanes in CAT(0) cube complexes. Two hyperplanes $\mathrm{H}$ and $\mathrm{K}$ intersect if their geometric realizations intersect, or equivalently if there is a square $\{x, y, z, w\}$ such that $\{x, y\}$ and $\{z, w\}$ belong to $H$ while $\{x, z\}$ and $\{y, w\}$ belong to $K$.

2.5. Lemma ([NR98a], Prop. 2.10). A hyperplane in a CAT(0) cube complex does not selfintersect. In fact if a vertex $\mathrm{x}$ is adjacent to a hyperplane $\mathrm{H}$, then there is a unique edge $\{x, \mathrm{y}\}$ which crosses $\mathrm{H}$. 
We shall use the notation $y=x^{\text {op }}$ for the vertex $y$ opposite $x$ across $H$ (obviously this depends on the choice of $\mathrm{H}$ ).

Proof of Proposition 2.4 By the Proposition 2.3 it suffices to show that any edge-path which is not a geodesic can be reduced to an edge-path of shorter length by a sequence of corner moves and simple cancellations. Assume, for the sake of a contradiction, that not every non-geodesic edge-path is so reducible and let $v_{0}, \ldots, v_{n}$ be an non-geodesic edge-path of minimal length that is not reducible. Since it is not a geodesic it must cross some hyperplane $\mathrm{H}$ twice. By minimality, it must do so between $v_{0}$ and $v_{1}$, and then again between $v_{n-1}$ and $v_{n}$. Moreover the edge-path $v_{1}, \ldots, v_{n-1}$ must be a geodesic. Therefore by [Sag95, Thm. 4.13], all the vertices $v_{2}, \ldots, v_{n-1}$ are adjacent to $H$. If $n=2$, then both $v_{0}$ and $v_{n}$ are opposite $v_{1}$ across $H$, and so $v_{0}=v_{n}$ by Lemma 2.5. The path $v_{0}, v_{1}, v_{2}$ is therefore $v_{0}, v_{1}, v_{0}$ and hence is reducible by a simple cancellation, which is a contradiction. If $n>2$, then by [Sag95, Thm. 4.12] the subset $\left\{v_{0}, v_{1}, v_{2}, v_{2}^{\text {op }}\right\}$ is a square. The path $v_{0}, v_{2}^{\text {op }}, v_{2}, \ldots, v_{n}$ is obtained from $v_{0}, \ldots, v_{n}$ by a corner move. But the path $v_{2}^{\text {op }}, v_{2}, \ldots v_{n}$ has length $n-1$ and is not a geodesic (it crosses $\mathrm{H}$ twice). It is therefore reducible by corner moves and simple cancellations. It follows that the path $v_{0}, v_{2}^{\text {op }}, v_{2}, \ldots, v_{n}$ is reducible as well, and hence so is $v_{0}, \ldots, v_{n}$, which is again a contradiction.

The convex hull $\mathfrak{C}(S)$ of a set $S$ of vertices in $X$ is the intersection of all the half-spaces that contain S. A set is convex if it is equal to its convex hull. It follows from Proposition 2.1 that every convex set is geodesically convex in the sense that it contains every geodesic between any two of its points. In the case where $S=\{x, y\}$, the convex hull is usually called the interval from $x$ to $y$ and thanks to Corollary 2.2 it can alternately be characterized as the set of all points that lie on geodesics from $x$ to $y$.

In Section 5 we shall need the following technical result:

2.6. Lemma. Let $x$ and $y$ be vertices in a CAT(0) cube complex and let $\mathrm{b}$ be a vertex of some cube $\mathrm{C}$ that also contains $\mathrm{y}$ as a vertex. There is a vertex $\mathrm{c}$ of $\mathrm{C}$ such that $\mathfrak{C}(\mathrm{x}, \mathrm{y}, \mathrm{b}) \subseteq \mathfrak{C}(\mathrm{x}, \mathrm{c})$.

Proof. Let $\mathfrak{H}$ be the set of all hyperplanes, necessarily passing through the cube $C$, that separate $b$ from $y$ but not $x$ from $y$. Let $c$ be the vertex of $C$ for which $\mathfrak{H}(y, c)=\mathfrak{H}$. We shall show that

$$
\mathfrak{C}(x, y, b) \subseteq \mathfrak{C}(x, c)
$$

To do so, we must show that $y \in \mathfrak{C}(x, c)$ and $b \in \mathfrak{C}(x, c)$. By construction of the vertex $c$, $\mathfrak{H}(x, y)$ is disjoint from $\mathfrak{H}(y, c)$. It therefore follows from Corollary 2.2 that $y$ lies on a geodesic from $x$ to $c$ and hence that $y \in \mathfrak{C}(x, c)$. Similarly, the definition of $\mathfrak{H}=\mathfrak{H}(x, c)$ asserts that $\mathfrak{H}(y, c)=\mathfrak{H}(b, c) \backslash \mathfrak{H}(x, y)$, and therefore

$$
\mathfrak{H}(b, c) \subseteq \mathfrak{H}(x, y) \cup \mathfrak{H}(y, c) .
$$

Since $y$ lies on a geodesic from $x$ to $c$, the union is $\mathfrak{H}(x, c)$. Therefore $\mathfrak{H}(b, c) \subseteq \mathfrak{H}(x, c)$, and so $\mathrm{b}$ lies on a geodesic from $x$ to $\mathrm{c}$, and hence is in the interval from $x$ to $\mathrm{c}$.

The next three results have to do with multiple hyperplanes that are adjacent to a single vertex. 
2.7. Proposition ([NR98a], Lemma 2.14). If two hyperplanes in a CAT (0) cube complex intersect and are both adjacent to the same vertex, then they intersect in a square containing that vertex.

2.8. Proposition. Let $\mathrm{H}_{1}, \ldots, \mathrm{H}_{\mathrm{n}}$ be hyperplanes in a CAT(0) cube complex, all adjacent to a vertex $\mathrm{x}$. If there is a vertex $\mathrm{y}$ in $\mathrm{X}$ such that each hyperplane $\mathrm{H}_{\mathrm{i}}$ separates $\mathrm{x}$ from $\mathrm{y}$, then there is a cube of dimension $\mathrm{n}$ in which all the hyperplanes $\mathrm{H}_{i}$ intersect.

Proof. This follows from Proposition 3.3 in [NR98a] (compare the remark following the proof of that proposition).

2.9. Proposition. Let $\mathrm{x}$ and $\mathrm{y}$ be vertices of $\mathrm{CAT}(0)$ cube complex. There exists a geodesic path from $\mathrm{x}$ to $\mathrm{y}$ that crosses all the hyperplanes in $\mathfrak{H}(\mathrm{x}, \mathrm{y})$ that are adjacent to $\mathrm{x}$ before it crosses any other hyperplane.

Proof. By Proposition 2.8, there is a cube $C$ of dimension $n$ containing $x$ as a vertex in which the $n$ hyperplanes in $\mathfrak{H}(x, y)$ that are adjacent to $x$ intersect. Let $v$ be the vertex diagonally across $C$ from $x$. By the equivalence of (a) and (e) in Corollary 2.2, there is a geodesic from $x$ to $y$ that passes through $v$, and any such geodesic has the required property.

Finally, two hyperplanes are parallel if they do not intersect.

2.10. Lemma. Two parallel hyperplanes separate a $\mathrm{CAT}(0)$ cube complex into at most three components.

Proof. It is convenient to work in the geometric realization. Let $\mathrm{H}$ and $\mathrm{K}$ be parallel hyperplanes. Since $\mathrm{K}$ is connected, it is contained entirely in one component $\mathrm{H}_{+}$of the complement of $\mathrm{H}$. It follows that one of $\mathrm{K}_{+}$or $\mathrm{K}_{-}$does not intersect $\mathrm{H}_{-}$, for if both were to intersect $\mathrm{H}_{-}$, then there would be a geodesic between a point in $\mathrm{K}_{+}$and a point in $\mathrm{K}_{-}$within $\mathrm{H}_{-}$, and hence a point of $\mathrm{K}$ within $\mathrm{H}_{-}$. If say $\mathrm{K}_{-} \cap \mathrm{H}_{-}=\emptyset$, then

$$
\begin{aligned}
\mathrm{X} \backslash(\mathrm{H} \cup \mathrm{K}) & =\left(\mathrm{H}_{+} \cup \mathrm{H}_{-}\right) \cap\left(\mathrm{K}_{+} \cup \mathrm{K}_{-}\right) \\
& =\left(\mathrm{H}_{+} \cap \mathrm{K}_{+}\right) \cup\left(\mathrm{H}_{+} \cap \mathrm{K}_{-}\right) \cup\left(\mathrm{H}_{-} \cap \mathrm{K}_{+}\right),
\end{aligned}
$$

as required.

\section{Cocycles From Cube Complexes}

An action of a group on a cube complex $X$ is an action on the set of vertices of $X$ that maps cubes to cubes. In this section we shall associate to the action of a group $G$ on a CAT $(0)$ cube complex $X$ a holomorphic family of cocycles $c_{z}: X \times X \rightarrow \mathcal{B}\left(\ell^{2}(X)\right)$ parametrized by $z \in \mathbb{D}$.

As in Section 1, we associate to each $z \in \mathbb{D}$ a complex number $w \in \mathbb{C}$ satisfying $z^{2}+w^{2}=1$ in such a way that $w$ depends holomorphically on $z$. Let $x$ and $y$ be adjacent vertices in $X$ and let $\mathrm{H}$ be the hyperplane that separates them, oriented so that $x \in \mathrm{H}_{+}$and $y \in \mathrm{H}_{-}$. Let us denote by

\footnotetext{
${ }^{2}$ In fact exactly three.
} 
$\partial \mathrm{H}_{+}$the set of vertices adjacent to $\mathrm{H}$ that lie in $\mathrm{H}_{+}$, and by $\partial \mathrm{H}_{-}$the set of vertices adjacent to $\mathrm{H}$ that lie in $\mathrm{H}_{-}$. Define a bounded operator $c_{z}(x, y)$ on $\ell^{2}(X)$ by means of the formula

$$
c_{z}(x, y) \delta_{v}= \begin{cases}w \delta_{v}-z \delta_{v^{\mathrm{op}}} & v \in \partial \mathrm{H}_{+} \\ w \delta_{v}+z \delta_{v^{\mathrm{op}}} & v \in \partial \mathrm{H}_{-} \\ \delta_{v} & v \notin \partial \mathrm{H}_{+} \cup \partial \mathrm{H}_{-}\end{cases}
$$

(where $v^{\text {op }}$ denotes the vertex adjacent to $v$ across $\mathrm{H}$ ). The definition is consistent with the one we made for trees in Section 1. However in the present, more general situation $c_{z}(x, y)$ is potentially nontrivial on many more basis vectors than $\delta_{x}$ and $\delta_{y}$ alone: it is nontrivial on the basis vector $\delta_{v}$ whenever $v$ is adjacent to the hyperplane $H$.

Recall that a subspace of a Hilbert space is a reducing subspace for an operator $\mathrm{T}$ if and only if both the subspace and its orthogonal complement are invariant under $\mathrm{T}$. If the vertices $v$ and $v^{\text {op }}$ are adjacent across $H$, then the two-dimensional subspace spanned by $\delta_{v}$ and $\delta_{v^{\text {op }}}$ is reducing for $c_{z}(x, y)$, as is the subspace spanned by all $\delta_{v}$ for which $v$ is not adjacent to $v$. Thus by Lemma 2.5 , $c_{z}(x, y)$ is the direct sum of a family of operators on two-dimensional subspaces together with the identity operator on the joint orthogonal complement of these two-dimensional subspaces. On the two-dimensional subspace spanned by the ordered pair $\left(\delta_{v}, \delta_{v^{\text {op }}}\right)$, where $v \in \partial \mathrm{H}_{+}$, the operator $c_{z}(x, y)$ is given by the matrix

$$
\left(\begin{array}{cc}
w & z \\
-z & w
\end{array}\right)
$$

3.1. Lemma. For every pair of adjacent edges $x$ and $y$, and for every $z \in \mathbb{D}, c_{z}(x, y) c_{z}(y, z)=I$.

Proof. This follows from the fact that the inverse of the matrix (3.2) is the transpose of (3.2).

If $x$ and $y$ are any two vertices in $X$, then we should like to define

$$
c_{z}(x, y)=c_{z}\left(v_{0}, v_{1}\right) c_{z}\left(v_{1}, v_{2}\right) \cdots c_{z}\left(v_{n-1}, v_{n}\right)
$$

where $x=v_{0}, \ldots, v_{n}=y$ are the vertices in an edge-path from $x$ to $y$. In the case of trees, Lemma 3.1 is sufficient to prove that the product (3.3) is independent of the choice of path from $x$ to $y$. For general CAT $(0)$ cube complexes we need the following additional computation.

3.2. Lemma. Let $\mathrm{x}, v, \mathrm{y}$ and $\mathrm{x}, v^{\prime}, \mathrm{y}$ be edge-paths of length two from $\mathrm{x}$ to $\mathrm{y}$. If the four vertices $x, v, v^{\prime}, y$ span a square in the cube complex $X$, then $c_{z}(x, v) c_{z}(v, y)=c_{z}\left(x, v^{\prime}\right) c_{z}\left(v^{\prime}, y\right)$. 
Proof. The relation between the four points is depicted in the following diagram:

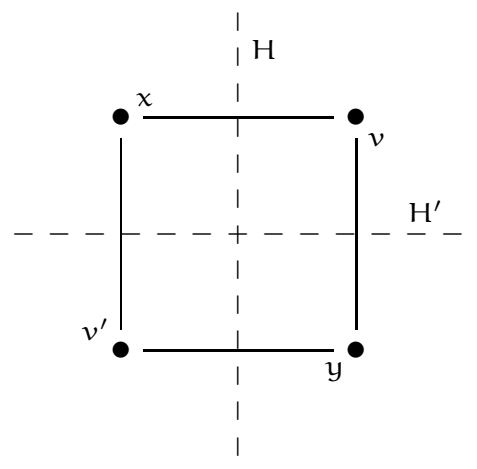

The hyperplane $\mathrm{H}$ separating $\mathrm{x}$ and $v$ also separates $v^{\prime}$ and $y$, while the hyperplane $\mathrm{H}^{\prime}$ separating $x$ and $v^{\prime}$ also separates $v$ and $y$. As result $c_{z}(x, v)=c_{z}\left(v^{\prime}, y\right)$ and $c_{z}\left(x, v^{\prime}\right)=c_{z}(v, y)$, since the definition of $c_{z}$ for a pair of adjacent vertices depends only on the oriented hyperplane separating them. So our goal is to show that

$$
c_{z}(x, v) c_{z}\left(x, v^{\prime}\right)=c_{z}\left(x, v^{\prime}\right) c_{z}(x, v) .
$$

By Lemmas 2.5 and 2.7 the set $X$ can be written as a disjoint union of:

(a) Four-element sets consisting of the vertices of a square in which $\mathrm{H}$ and $\mathrm{H}^{\prime}$ intersect.

(b) Two-element sets consisting of vertices adjacent across $\mathrm{H}$ but with neither adjacent to $\mathrm{H}^{\prime}$.

(c) Two-element sets consisting of vertices adjacent across $\mathrm{H}^{\prime}$ but with neither adjacent to $\mathrm{H}$.

(d) Single-element sets consisting of a vertex adjacent to neither $\mathrm{H}$ nor $\mathrm{H}^{\prime}$.

The corresponding subspaces of $\ell^{2}(X)$ are reducing for $c_{z}(x, v)$ and $c_{z}\left(x, v^{\prime}\right)$. On the four-dimensional subspaces, the prototype of which is the one spanned by $\delta_{x}, \delta_{v}, \delta_{v^{\prime}}, \delta_{y}$, the operators $c_{z}(x, v)$ and $\mathrm{c}_{z}\left(\chi, v^{\prime}\right)$ are represented by matrices

$$
\left(\begin{array}{cccc}
w & z & 0 & 0 \\
-z & w & 0 & 0 \\
0 & 0 & w & z \\
0 & 0 & -z & w
\end{array}\right) \text { and }\left(\begin{array}{cccc}
w & 0 & z & 0 \\
0 & w & 0 & z \\
-z & 0 & w & 0 \\
0 & -z & 0 & w
\end{array}\right)
$$

and these two matrices commute. On the remaining subspaces, either one or both of $c_{z}(x, v)$ and $c_{z}\left(x, v^{\prime}\right)$ acts as the identity, and so the two operators commute there too.

3.3. Lemma. The expression (3.3) defining $\mathrm{c}(x, y)$ for general $x, y \in X$ is independent of the edge-path $v_{0}, v_{1}, \ldots, v_{\mathrm{n}}$ connecting $x$ to $\mathrm{y}$.

Proof. According to Proposition 2.4 any two paths connecting $x$ to $y$ are related by a sequence of corner moves and simple cancellations. Lemma 3.1 shows that simple cancellations do not alter (3.3). Lemma 3.2 shows that corner moves do not alter (3.3) either. 
3.4. Proposition. Let $\mathrm{X}$ be a $\mathrm{CAT}(0)$ cube complex and let $\mathrm{G}$ be a discrete group acting on $\mathrm{X}$. For every $z \in \mathbb{D}$ the function $c_{z}: X \times X \rightarrow \mathcal{B}\left(\ell^{2}(X)\right)$ defined by (3.3) is a cocycle for the permutation representation of $\mathrm{G}$ on $\ell^{2}(\mathrm{X})$.

Fix $x \in X$. Thanks to the proposition we can construct representations $\pi_{z}(g)=c_{z}(x, g x) \pi(g)$ of the group $\mathrm{G}$ into the bounded invertible operators on $\ell^{2}(X)$.

3.5. Proposition. The family $\left\{\pi_{z}\right\}_{z \in \mathbb{D}}$ is a holomorphic family of representations of $\mathrm{G}$. If $z \in \mathbb{D}$ is real, then the representation $\pi_{z}$ is unitary.

3.6. Proposition. The matrix coefficient $\left\langle\pi_{z}(g) \delta_{x}, \delta_{x}\right\rangle$ is equal to $z^{\mathrm{d}(x, g x)}$.

Proof. By definition, $\left\langle\pi_{z}(g) \delta_{x}, \delta_{x}\right\rangle=\left\langle c_{z}(x, g x) \delta_{g x}, \delta_{x}\right\rangle$, so it suffices to prove that

$$
\left\langle c_{z}(x, y) \delta_{y}, \delta_{x}\right\rangle=z^{d(x, y)}
$$

for every $x$ and $y$ in $X$. Note first that by definition $c_{z}(x, y)$ can be written as a product of $d(x, y)$ many operators $T=c_{z}\left(v_{i}, v_{i+1}\right)$, each of which has the property that $\left\langle T \delta_{a}, \delta_{b}\right\rangle=0$ if $d(a, b)>1$. It follows that

$$
\left\langle c_{z}(x, y) \delta_{a}, \delta_{b}\right\rangle=0 \text { if } d(a, b)>d(x, y) .
$$

We can now prove the formula (3.4) by induction on $d(x, y)$. Let $x=v_{0}, v_{1}, \ldots, v_{n}=y$ be a geodesic edge-path from $x$ to $y$. Using (3.5) and the induction hypothesis we have that

$$
c_{z}\left(v_{1}, v_{n}\right) \delta_{v_{n}}=0 \cdot \delta_{v_{0}}+z^{n-1} \delta_{v_{1}}+\text { terms orthogonal to } \delta_{v_{0}}, \delta_{v_{1}} .
$$

Using the explicit formula for $c_{z}\left(v_{0}, v_{1}\right)$ we get that

$$
c_{z}\left(v_{0}, v_{n}\right) \delta_{v_{n}}=c_{z}\left(v_{0}, v_{1}\right) c_{z}\left(v_{1}, v_{n}\right) \delta_{v_{n}}=z^{n} \delta_{v_{0}}+\text { terms orthogonal to } \delta_{v_{0}},
$$

as required.

\section{Calculation of Matrix Coefficients}

The remainder of the paper will be devoted to proving that the cocycle $c_{z}$ is uniformly bounded. Following the approach we took for trees in Section 1, we shall begin by studying the individual matrix coefficients of the operator $c_{z}(x, y)$. Define the matrix coefficient $c_{a b}$ by the formula

$$
c_{z}(x, y) \delta_{b}=\sum_{a \in X} c_{a b} \delta_{a}
$$

or equivalently $c_{a b}=\left\langle c_{z}(x, y) \delta_{b}, \delta_{a}\right\rangle$.

4.1. Lemma. Let $\mathrm{x}$ and $\mathrm{y}$ be any two vertices in $\mathrm{X}$. If $\mathrm{K}$ is a hyperplane which does not separate $x$ from $\mathrm{y}$, then the subspaces $\ell^{2}\left(\mathrm{~K}_{ \pm}\right)$are reducing subspaces for $\mathrm{c}_{z}(\mathrm{x}, \mathrm{y})$. Hence if $\mathrm{c}_{\mathrm{ab}}$ is non-zero for some $z \in \mathbb{D}$, then $\mathfrak{H}(\mathrm{a}, \mathrm{b}) \subseteq \mathfrak{H}(x, y)$. 
Proof. Assume first that $x$ and $y$ are adjacent. The only nonzero matrix coefficients of $c_{z}(x, y)$ apart from those on the diagonal are those $c_{a b}$ for which $a$ and $b$ are adjacent to one another across the hyperplane $H$ separating $x$ from $y$. So if $c_{a b}$ is nonzero for some $z \in \mathbb{D}$, then $a$ and $b$ must lie on opposite sides of $\mathrm{H}$, and hence on the same side of $\mathrm{K}$. In general, let $v_{0}, \ldots, v_{n}$ be a geodesic edge-path from $x$ to $y$, so that

$$
c_{z}(x, y)=c_{z}\left(v_{0}, v_{1}\right) c_{z}\left(v_{1}, v_{2}\right) \cdots c_{z}\left(v_{n-1}, v_{n}\right) .
$$

The hyperplane $\mathrm{K}$ separates no $v_{i}$ from $v_{i+1}$. Therefore the subspaces $\ell^{2}\left(\mathrm{~K}_{ \pm}\right)$are reducing for each $c_{z}\left(v_{i-1}, v_{i}\right)$, and hence for $c_{z}(x, y)$ as well.

4.2. Proposition. Let $\mathrm{x}, \mathrm{y}$ and $\mathrm{b}$ be vertices of $\mathrm{X}$. If $\mathrm{c}_{\mathrm{ab}}$ is nonzero for some $z \in \mathbb{D}$, then $\mathrm{a}$ lies in the convex hull of $\{x, y, b\}$.

Proof. Assume that $\mathrm{c}_{\mathrm{ab}}$ is nonzero for some $z \in \mathbb{D}$. Let $\mathrm{H}$ be a hyperplane for which all of $x$, $y$ and $b$ lie in the same half-space. If $a$ lies in the other half-space then $H \in \mathfrak{H}(a, b)$ but not in $\mathfrak{H}(x, y)$ contradicting Lemma 4.1 .

4.3. Definition. Let $x, y \in X$. A geodesic order on $\mathfrak{H}(x, y)$ is a linear order for which there exists a geodesic edge-path from $x$ to $y$ such that $\mathrm{H}^{\prime}<\mathrm{H}^{\prime \prime}$ if and only if the path crosses $\mathrm{H}^{\prime}$ before it crosses $\mathrm{H}^{\prime \prime}$.

4.4. Lemma. If $\mathrm{c}_{\mathrm{ab}}$ is nonzero for some $z \in \mathbb{D}$, then every geodesic order on $\mathfrak{H}(x, y)$ induces a geodesic order on $\mathfrak{H}(\mathrm{a}, \mathrm{b})$.

Proof. We shall prove the lemma by induction on the integer $d(x, y)$ (starting with $d(x, y)=0$, where the result is trivial). Assume that $c_{a b} \neq 0$, for some $z \in \mathbb{D}$. Let $\left\{H_{1}, \ldots, H_{n}\right\}$ be a geodesic order on $\mathfrak{H}(x, y)$ and let $v_{0}, \ldots, v_{n}$ be the corresponding geodesic path from $x$ to $y$, so that

$$
c_{z}(x, y)=c_{z}\left(v_{0}, v_{1}\right) c_{z}\left(v_{1}, v_{2}\right) \cdots c_{z}\left(v_{n-1}, v_{n}\right)=c_{z}\left(v_{0}, v_{1}\right) c_{z}\left(v_{1}, v_{n}\right) .
$$

To prove that the given geodesic order on $\mathfrak{H}(x, y)$ restricts to a geodesic order on $\mathfrak{H}(a, b)$ we shall consider two cases. The first is that $a$ is not adjacent to the hyperplane $H_{1}$ that separates $v_{0}$ from $v_{1}$. In this case, since

$$
c_{a b}=\left\langle c_{z}\left(v_{1}, v_{n}\right) \delta_{b}, c_{z}\left(v_{0}, v_{1}\right)^{*} \delta_{a}\right\rangle,
$$

and since $c_{z}\left(v_{0}, v_{1}\right)^{*} \delta_{a}=\delta_{a}$, the ab-matrix coefficient for $c_{z}(x, y)$ is equal to the ab-matrix coefficient for $c_{z}\left(v_{1}, v_{n}\right)$ and in particular the latter is nonzero. By the induction hypothesis, the given geodesic order on $\mathfrak{H}\left(v_{1}, v_{n}\right)$ restricts to a geodesic order on $\mathfrak{H}(a, b)$. But this order on $\mathfrak{H}(a, b)$ is the same as the order restricted from $\mathfrak{H}(x, y)$.

In the second case, $a$ is adjacent to $\mathrm{H}_{1}$. Denote by $\mathrm{a}^{\text {op }}$ the vertex adjacent to $a$ across $\mathrm{H}_{1}$. From (4.1) and the definition of $c_{z}\left(v_{0}, v_{1}\right)$ we get that

$$
c_{a b}=w \cdot\left\langle c_{z}\left(v_{1}, v_{n}\right) \delta_{b}, \delta_{a}\right\rangle \pm z \cdot\left\langle c_{z}\left(v_{1}, v_{n}\right) \delta_{b}, \delta_{a^{o p}}\right\rangle .
$$

If $\mathrm{H}_{1}$ separates $a$ from $b$ then by Lemma 4.1 the first inner product in (4.2) is zero, and hence

$$
c_{\mathrm{ab}}= \pm z \cdot\left\langle c_{z}\left(v_{1}, v_{\mathrm{n}}\right) \delta_{\mathrm{b}}, \delta_{\mathrm{a}^{\mathrm{op}}}\right\rangle \text {. }
$$


Therefore the $a^{\text {op }} b$-matrix coefficient for $c_{z}\left(v_{1}, v_{n}\right)$ is nonzero. By the induction hypothesis, the given geodesic order on $\mathfrak{H}\left(v_{1}, v_{n}\right)$ restricts to a geodesic order on $\mathfrak{H}\left(\mathrm{a}^{\mathrm{op}}, \mathrm{b}\right)$. Since

$$
\mathfrak{H}(a, b)=\left\{\mathrm{H}_{1}\right\} \cup \mathfrak{H}\left(\mathrm{a}^{\mathrm{op}}, \mathrm{b}\right) \quad \text { and } \quad \mathfrak{H}(x, y)=\left\{\mathrm{H}_{1}\right\} \cup \mathfrak{H}\left(v_{1}, v_{n}\right),
$$

it follows easily that the geodesic order on $\mathfrak{H}(x, y)$ restricts to a geodesic order on $\mathfrak{H}(a, b)$. If $\mathrm{H}_{1}$ does not separate $a$ from $b$, then it separates $a^{\text {op }}$ from $b$. The second inner product in (4.2) is therefore zero, and hence

$$
c_{a b}=w \cdot\left\langle c_{z}\left(v_{1}, v_{n}\right) \delta_{b}, \delta_{a}\right\rangle
$$

so that $a b$-matrix coefficient for $c_{z}\left(v_{1}, v_{n}\right)$ is nonzero. By the induction hypothesis, the geodesic order on $\mathfrak{H}\left(v_{1}, v_{n}\right)$ restricts to a geodesic order on $\mathfrak{H}(a, b)$. This immediately implies that the given geodesic order on $\mathfrak{H}(x, y)$ restricts to a geodesic order on $\mathfrak{H}(a, b)$.

4.5. Lemma. A linear ordering $\left\{\mathrm{H}_{1}, \ldots, \mathrm{H}_{n}\right\}$ on $\mathfrak{H}(x, y)$ is a geodesic ordering if and only if the vertex $v_{0}=x$ is adjacent to $\mathrm{H}_{1}$ and for each $\mathrm{i}=1, \ldots, \mathrm{n}$ the vertex $v_{\mathrm{i}}$ obtained by successively reflecting $v_{0}$ across $\mathrm{H}_{1}, \ldots, \mathrm{H}_{i-1}$ is adjacent to $\mathrm{H}_{i}$. In this case the sequence of vertices $v_{0}, \ldots, v_{n}$ is a geodesic edge-path from $\mathrm{x}$ to $\mathrm{y}$.

Proof. If a linear ordering on $\mathfrak{H}(x, y)$ is induced from a geodesic edge-path from $x$ to $y$, then the sequence $v_{0}, \ldots, v_{n}$ is precisely the sequence of vertices along the path, and so the adjacency condition is satisfied. Conversely, if the adjacency condition is satisfied, then $v_{0}, \ldots, v_{n}$ is a geodesic edge-path from $x$ to $v_{n}$. The vertex $v_{n}$ must equal $y$ since any hyperplane $K$ separating the two points would separate either $v_{n}$ from $v_{0}=x$ or separate $x$ from $y$. In fact by construction of the path $v_{0}, \ldots, v_{n}$, the hyperplane $\mathrm{K}$ would necessarily separate both $v_{n}$ and $y$ from $v_{0}=x$, which is a contradiction since $K$ could not then separate $v_{n}$ from $y$.

4.6. Lemma. Let $\mathrm{x}, \mathrm{y}, \mathrm{a}, \mathrm{b} \in \mathrm{X}$ and suppose that no hyperplane in $\mathfrak{H}(\mathrm{x}, \mathrm{y})$ separates $\mathrm{b}$ from $\mathrm{a}$. Then $\left\langle\mathrm{c}_{z}(x, y) \delta_{b}, \delta_{\mathrm{a}}\right\rangle=w^{\ell}\left\langle\delta_{b}, \delta_{\mathrm{a}}\right\rangle$, where $\ell$ is the number of hyperplanes in $\mathfrak{H}(x, y)$ that are adjacent to $\mathrm{a}$.

Proof. The proof is by induction on $n=d(x, y)$. The case $n=0$ is trivial, so assume that $n>0$. Let $v_{0}, \ldots, v_{n}$ be a geodesic edge-path from $x$ to $y$ and let $\mathrm{H}$ be the hyperplane that separates $v_{n-1}$ from $v_{n}$. Then

$$
c_{z}\left(v_{n-1}, v_{n}\right) \delta_{b}= \begin{cases}w \delta_{b} \pm z \delta_{b^{\text {op }}} & \text { if } \mathrm{b} \text { is adjacent to } \mathrm{H} \\ \delta_{\mathrm{b}} & \text { otherwise, }\end{cases}
$$

where $b^{\text {op }}$ is the vertex adjacent to $b$ across $H$. Since $\ell^{2}\left(H_{ \pm}\right)$are reducing subspaces for $c_{z}\left(v_{0}, v_{n-1}\right)$, it follows that $c_{z}\left(v_{0}, v_{n-1}\right) \delta_{b^{\text {op }}}$ is orthogonal to $\delta_{a}$. As a result

$$
\begin{aligned}
\left\langle c_{z}\left(v_{0}, v_{n}\right) \delta_{b}, \delta_{a}\right\rangle & =\left\langle c_{z}\left(v_{0}, v_{n-1}\right) c_{z}\left(v_{n-1}, v_{n}\right) \delta_{b}, \delta_{a}\right\rangle \\
& = \begin{cases}w\left\langle c_{z}\left(v_{0}, v_{n-1}\right) \delta_{b}, \delta_{a}\right\rangle & \text { if } b \text { is adjacent to } H \\
\left\langle c_{z}\left(v_{0}, v_{n-1}\right) \delta_{b}, \delta_{a}\right\rangle & \text { otherwise. }\end{cases}
\end{aligned}
$$

The result follows. 
Suppose that $c_{a b}$ is non-zero for some $z \in \mathbb{D}$. Fix a geodesic order $\left\{H_{1}, \ldots, H_{n}\right\}$ on $\mathfrak{H}(x, y)$, and let $v_{0}, \ldots, v_{n}$ be the corresponding geodesic edge-path from $x$ to $y$. Let

$$
\mathfrak{H}(\mathrm{a}, \mathrm{b})=\left\{\mathrm{H}_{\mathrm{n}_{1}}, \ldots, \mathrm{H}_{\mathrm{n}_{\mathrm{p}}}\right\}
$$

and let $a_{0}, \ldots, a_{p}$ be the geodesic edge-path from $a$ to $b$, guaranteed by Lemma 4.4, that corresponds to this ordering of $\mathfrak{H}(a, b)$. Note that $d(a, b)=p$.

For $j=0, \ldots, p$, let $\ell_{j}$ be the number of hyperplanes in $\left\{H_{k}: n_{j}<k<n_{j+1}\right\}$ that are adjacent to $a_{j}$ (for convenience we are setting $n_{0}=0$ and $n_{p+1}=n+1$ ).

4.7. Lemma. With the above notation, $\mathrm{c}_{\mathrm{ab}}= \pm z^{\mathrm{p}} \mathcal{W}^{\ell_{0}+\cdots+\ell_{\mathrm{p}}}$.

Proof. We shall prove by induction on $j$, from $j=p$ down to $j=0$, that

$$
\left\langle\mathrm{c}_{z}\left(v_{n_{j}}, v_{n}\right) \delta_{b}, \delta_{a_{j}}\right\rangle= \pm z^{p-j} w^{\ell_{j}+\cdots+\ell_{p}} .
$$

The case $j=p$ is a consequence of Lemma 4.6, while the assertion in the current lemma is the case $j=0$. Assume that (4.3) holds for a given $j$. To compute (4.3) with $j-1$ in place of $j$ we shall write

$$
c_{z}\left(v_{n_{j-1}}, v_{n}\right) \delta_{b}=c_{z}\left(v_{n_{j-1}}, v_{n_{j}-1}\right) c_{z}\left(v_{n_{j}-1}, v_{n}\right) \delta_{b}
$$

and then write $c_{z}\left(v_{n_{j}-1}, v_{n_{p+1}}\right) \delta_{b}$ as a finite linear combination

$$
c_{z}\left(v_{n_{j}-1}, v_{n}\right) \delta_{b}=\sum \alpha_{k} \delta_{b_{k}}
$$

As long as the sum contains no zero terms, it follows from Lemma 4.1 that every vertex $b_{k}$ lies in the same half-space of every $H \in \mathfrak{H}\left(v_{n_{j-1}}, v_{n_{j}-1}\right)$ as the vertex $b$. Moreover $b$ and $a_{j-1}$ lie in the same half-spaces of these hyperplanes because the only hyperplanes in $\mathfrak{H}(x, y)$ that separate $a_{j-1}$ from $b=a_{p}$ are $H_{n_{j}}, \ldots, H_{n_{p}}$. It therefore follows from Lemma 4.6 that

$$
\begin{aligned}
\left\langle c_{z}\left(v_{n_{j-1}}, v_{n}\right) \delta_{b}, \delta_{a_{j-1}}\right\rangle & =\sum \alpha_{k}\left\langle c_{z}\left(v_{n_{j-1}}, v_{n_{j}-1}\right) \delta_{b_{k}}, \delta_{a_{j-1}}\right\rangle \\
& =w^{\ell_{j-1}} \sum \alpha_{k}\left\langle\delta_{b_{k}}, \delta_{a_{j-1}}\right\rangle \\
& =w^{\ell_{j-1}}\left\langle c_{z}\left(v_{n_{j}-1}, v_{n}\right) \delta_{b}, \delta_{a_{j-1}}\right\rangle .
\end{aligned}
$$

In addition,

$$
\left\langle c_{z}\left(v_{n_{j}-1}, v_{n}\right) \delta_{b}, \delta_{a_{j-1}}\right\rangle=\left\langle c_{z}\left(v_{n_{j}}, v_{n}\right) \delta_{b}, c_{z}\left(v_{n_{j}-1}, v_{n_{j}}\right)^{*} \delta_{a_{j-1}}\right\rangle
$$

and

$$
\left\langle c_{z}\left(v_{n_{j}}, v_{n}\right) \delta_{b}, c_{z}\left(v_{n_{j}-1}, v_{n_{j}}\right)^{*} \delta_{a_{j-1}}\right\rangle= \pm\left\langle c_{z}\left(v_{n_{j}}, v_{n}\right) \delta_{b}, \bar{z} \delta_{a_{j}}\right\rangle,
$$

by definition of the geodesic path $a_{0}, \ldots, a_{p}$. We conclude that

$$
\left\langle c_{z}\left(v_{n_{j-1}}, v_{n}\right) \delta_{b}, \delta_{a_{j-1}}\right\rangle= \pm z^{p-(j-1)} w^{\ell_{j-1}+\cdots+\ell_{p}}
$$

as required.

4.8. Lemma. If $x, y, a, b \in X$, then $\mathfrak{H}(x, y) \backslash \mathfrak{H}(a, b) \subset \mathfrak{H}(a, x) \triangle \mathfrak{H}(b, y)$ (symmetric difference). 
Proof. Let $\mathrm{H} \in \mathfrak{H}(x, y) \backslash \mathfrak{H}(a, b)$. Then $x$ and $y$ are on opposite sides of $H$ while $a$ and $b$ are on the same side. Either $a$ and $b$ lie on the side containing $y$, in which case $H \in \mathfrak{H}(a, x) \backslash \mathfrak{H}(b, y)$ or they lie on the side containing $x$, in which case $H \in \mathfrak{H}(b, y) \backslash \mathfrak{H}(a, x)$.

4.9. Proposition. Let $\mathrm{x}$ and $\mathrm{y}$ be vertices of $\mathrm{X}$. As usual, for $\mathrm{a}, \mathrm{b} \in \mathrm{X}$ let $\mathrm{c}_{\mathrm{ab}}=\left\langle\mathrm{c}_{z}(\mathrm{x}, \mathrm{y}) \delta_{\mathrm{b}}, \delta_{\mathrm{a}}\right\rangle$. If $\mathrm{c}_{\mathrm{ab}}$ is nonzero for some $z \in \mathbb{D}$, then

$$
\mathrm{c}_{\mathrm{ab}}= \pm z^{\mathrm{d}(\mathrm{a}, \mathrm{b})} \mathcal{w}^{\ell}
$$

for some non-negative integer $\ell$ not exceeding twice the dimension of $\mathrm{X}$.

Proof. In view of Lemma 4.7 we need only show that the sum $\ell_{0}+\cdots+\ell_{p}$ appearing there is bounded by twice the dimension of $X$. Recall that $\ell_{j}$ is the cardinality of the set $\mathfrak{H}_{j}$ of hyperplanes in $\left\{H_{k}: n_{j}<k<n_{j+1}\right\}$ that are adjacent to $a_{j}$. The only hyperplanes in $\mathfrak{H}(x, y)$ that separate $a$ from $b$ are $H_{n_{1}}, \ldots, H_{n_{p}}$. Therefore

$$
\mathfrak{H}_{0} \cup \cdots \cup \mathfrak{H}_{p} \subseteq \mathfrak{H}(x, y) \backslash \mathfrak{H}(a, b)
$$

and so by Lemma 4.8 ,

$$
\mathfrak{H}_{0} \cup \cdots \cup \mathfrak{H}_{\mathrm{p}} \subseteq \mathfrak{H}(\mathrm{a}, x) \triangle \mathfrak{H}(\mathrm{b}, \mathrm{y}) .
$$

We shall show that if $H$ belongs to both $\mathfrak{H}_{0} \cup \cdots \cup \mathfrak{H}_{p}$ and $\mathfrak{H}(a, x) \backslash \mathfrak{H}(b, y)$, then $H$ is adjacent to the vertex a. Similarly, we shall show that if $H$ belongs to both $\mathfrak{H}_{0} \cup \cdots \cup \mathfrak{H}_{\mathrm{p}}$ and $\mathfrak{H}(b, y) \backslash \mathfrak{H}(a, x)$, then $H$ is adjacent to the vertex $b$. It will follow from this that $\mathfrak{H}_{0} \cup \cdots \cup \mathfrak{H}_{\mathrm{p}}$ is a union of two sets, the first consisting of hyperplanes adjacent to a that separate a from $x$, and the second consisting of hyperplanes adjacent to $b$ that separate $b$ from $y$. Since Proposition 2.8 implies that the hyperplanes in each set meet in a cube of $X$, it will follow that each set can have at most $\operatorname{dim}(X)$ elements, and therefore that $\ell_{0}+\cdots+\ell_{p}$ is bounded by $2 \operatorname{dim}(X)$, as required.

Let $\mathrm{H} \in \mathfrak{H}_{j}$. Thus $\mathrm{H}=\mathrm{H}_{k}$, where $n_{j}<k<n_{\mathfrak{j}+1}$, and $\mathrm{H}$ is adjacent to $a_{j}$. Assume in addition that $H \in \mathfrak{H}(a, x) \backslash \mathfrak{H}(b, y)$. We shall show that $a$ and indeed the entire geodesic edgepath $a_{0}, \ldots, a_{j}$ is adjacent to $H$ by proving that if a vertex $a_{s}$ on this path is adjacent to $H$, and if $s>1$, then $a_{s-1}$ is adjacent to $H$ too.

Let $\mathrm{K}$ be the hyperplane that separates $\mathrm{a}_{\mathrm{s}-1}$ from $\mathrm{a}_{\mathrm{s}}$ (thus $\mathrm{K}=\mathrm{H}_{\mathrm{n}_{\mathrm{s}}}$.) Orient the hyperplanes $\mathrm{H}$ and $\mathrm{K}$ so that $\mathrm{b} \in \mathrm{H}_{+} \cap \mathrm{K}_{+}$. Then $\mathrm{a} \in \mathrm{H}_{+} \cap \mathrm{K}_{-}$since $\mathrm{K} \in \mathfrak{H}(\mathrm{a}, \mathrm{b})$ while $\mathrm{H} \notin \mathfrak{H}(\mathrm{a}, \mathrm{b})$. In addition $y \in H_{+}$and $x \in H_{-}$since $H \notin \mathfrak{H}(b, y)$. Since $K<H$ in the geodesic order from $x$ to $y$, there is at least one vertex $v$ on the geodesic $v_{0}, \ldots, v_{n}$ that $\mathrm{K}$ but not $\mathrm{H}$ separates from $x$. If $x \in \mathrm{K}_{-}$, then $x \in \mathrm{H}_{-} \cap \mathrm{K}_{-}$and $v \in \mathrm{H}_{-} \cap \mathrm{K}_{+}$, while if $x \in \mathrm{K}_{+}$, then $x \in \mathrm{H}_{-} \cap \mathrm{K}_{+}$and $v \in \mathrm{H}_{-} \cap \mathrm{K}_{-}$. In either case, all four of the half-space intersections $\mathrm{H}_{ \pm} \cap \mathrm{K}_{ \pm}$are nonempty, and it therefore follows from Lemma 2.10 that $H$ and $K$ intersect. Since $a_{s}$ is adjacent to both $H$ and $K$, it follows from Proposition 2.7 that $H$ and $K$ intersect in a square having $a_{s}$ as a vertex, and this implies that $a_{s-1}$ is adjacent to $\mathrm{H}$, as required.

The proof that if $H$ belongs to both $\mathfrak{H}_{0} \cup \cdots \cup \mathfrak{H}_{p}$ and $\mathfrak{H}(b, y) \backslash \mathfrak{H}(a, x)$, then $H$ is adjacent to the vertex $b$ is exactly the same. 


\section{UNIFORM BOUNDEDNESS OF THE COCYCLE}

Our proof that the operators $c_{z}(x, y)$ are uniformly bounded as $x$ and $y$ range over all of $X$ (while $z$ ranges over a compact subset of $\mathbb{D}$ ) will be based on the results of the previous section and the following estimate of Chatterji and Ruane [CR05] (we shall offer our own proof of the estimate in the appendix).

5.1. Proposition. Let $\mathrm{X}$ be a finite-dimensional CAT( 0 ) cube complex and let $\mathrm{x}, \mathrm{y}$ be vertices of $\mathrm{X}$. If $\mathrm{k} \geq 0$, then denote by $\mathrm{B}(\mathrm{y}, \mathrm{k})$ the set of vertices of $\mathrm{X}$ of distance $\mathrm{k}$ or less to $\mathrm{y}$. Then

$$
\#(\mathfrak{C}(x, y) \cap B(y, k)) \leq(k+1)^{d}
$$

where $\mathrm{d}=\operatorname{dim}(X)$.

5.2. Lemma. Let $\mathrm{x}, \mathrm{y} \in \mathrm{X}$. There is a polynomial function $\mathrm{p}$ (depending only on the dimension of $\mathrm{X})$ such that for every $\mathrm{b} \in \mathrm{X}$ and every $\mathrm{k}$,

$$
\#\left\{a \in X: c_{a b}= \pm z^{k} w^{\ell} \text { for some } \ell \text { and all } z \in \mathbb{D}\right\} \leq p(k)
$$

and such that for every $\mathrm{a} \in \mathrm{X}$ and every $\mathrm{k}$,

$$
\#\left\{\mathrm{~b} \in \mathrm{X}: \mathrm{c}_{\mathrm{ab}}= \pm z^{\mathrm{k}} \mathrm{w}^{\ell} \text { for some } \ell \text { and all } z \in \mathbb{D}\right\} \leq \mathrm{p}(\mathrm{k})
$$

Proof. Fix $b \in X$. Let $x=u_{0}, \ldots, u_{m}=b$ be a geodesic edge-path from $x$ to $b$. Let $C$ be a cube containing the vertex $b$ in which meet all the hyperplanes that are adjacent to $b$ and that separate $b$ from $y$. Let $b=v_{0}, \ldots, v_{n}=y$ be a geodesic edge-path from $b$ to $y$ composed of $a$ path $v_{0}, \ldots, v_{i}$ consisting of vertices in $\mathrm{C}$, followed by a path $v_{i+1}, \ldots v_{n}$ which does not cross any hyperplane adjacent to $\mathrm{b}=v_{0}$ (see Proposition 2.9). By the cocycle property,

$$
c_{z}(x, y)=c_{z}\left(u_{0}, u_{1}\right) \cdots c_{z}\left(u_{m-1}, u_{m}\right) c_{z}\left(v_{0}, v_{1}\right) \cdots c_{z}\left(v_{n-1}, v_{n}\right) .
$$

Furthermore $c_{z}\left(v_{j}, v_{j+1}\right) \delta_{b}=\delta_{b}$ for all $j \geq i$ since $b$ is not adjacent to the hyperplane separating $v_{j}$ from $v_{j+1}$, and therefore $c_{z}(x, y) \delta_{b}=c_{z}\left(x, v_{i}\right) \delta_{b}$. Now, because $b$ and $v_{i}$ are vertices of the same cube $C$, it follows from Proposition 4.2 and Lemma 2.6 that there is another vertex $c$ of $C$ such that

$$
\left\{a \in X: c_{a b} \neq 0 \text { for some } z \in \mathbb{D}\right\} \subseteq \mathfrak{C}(x, c) .
$$

Note next that $d(b, c) \leq \operatorname{dim}(X)$, so that if $d(a, b)=k$, then $d(a, c) \leq k+\operatorname{dim}(X)$. In addition, if $c_{a b}= \pm z^{k} w^{\ell}$, then we proved in Proposition 4.9 that $k=d(a, b)$, from which it follows that $d(a, c) \leq k+\operatorname{dim}(X)$. Therefore

$$
\left\{a \in X: c_{a b}= \pm z^{k} w^{\ell} \text { for some } \ell \text { and all } z \in \mathbb{D}\right\} \subseteq \mathfrak{C}(x, c) \cap B(c, k+\operatorname{dim}(X))
$$

and Proposition 5.1 implies that for all $k$,

$$
\#\left\{a \in X: c_{a b}= \pm z^{k} w^{\ell} \text { for some } \ell \text { and all } z \in \mathbb{D}\right\} \leq(k+d+1)^{d},
$$

where $d=\operatorname{dim}(X)$.

To prove the second estimate, it suffices to note that $c_{z}(x, y)=c_{\bar{z}}(y, x)^{*}$, so that the ab-matrix coefficient of $c_{z}(x, y)$ is equal to the complex conjugate of the ba-matrix coefficient of $c_{\bar{z}}(y, x)$. 
Thus the second estimate, using the same polynomial $p(k)=(k+d+1)^{d}$, follows from the first.

Following the approach we took in Section 1, let us use the results of Section 4 to decompose the operator $c_{z}(x, y)$ as a linear combination

$$
c_{z}(x, y)=\sum_{k \geq 0} z^{k} c_{z}^{(k)}(x, y)
$$

in which the non-zero matrix coefficients of the operators $c_{z}^{(k)}(x, y)$ are all of the form $\pm w^{\ell}$, where $0 \leq \ell \leq 2 \operatorname{dim}(X)$. Note that this is actually a finite linear combination since, by Lemma 4.1, $c_{z}^{(k)}(x, y)=0$ when $k \geq d(x, y)$.

5.3. Proposition. For every compact subset $\mathrm{K} \subseteq \mathbb{D}$,

$$
\sup \left\{\left\|c_{z}(x, y)\right\|: x, y \in X, z \in K\right\}<\infty .
$$

Proof. Since $|w|^{2} \leq 2$, the matrix entries of $c_{z}^{(k)}(x, y)$ are all bounded in absolute value by $2^{\operatorname{dim}(X)}$. Furthermore there are at most $p(k)$ non-zero matrix entries in each row and column, where $p$ is the polynomial function of Lemma 5.2. Therefore, as in Section 1 ,

$$
\left\|c_{z}^{(k)}(x, y)\right\| \leq 2^{\operatorname{dim}(x)} p(k)
$$

It follows that

$$
\left\|c_{z}(x, y)\right\| \leq 2^{\operatorname{dim}(X)} \sum_{k=0}^{\infty}|z|^{k} p(k)
$$

and this gives the result.

Having established that the cocycle $c_{z}(x, y)$ is uniformly bounded, the proof of our main theorem is complete:

5.4. Definition. A CAT (0)-cubical group is a group $\mathrm{G}$ which admits an action on a finite-dimensional $\operatorname{CAT}(0)$ cube complex in such a way that $d(g x, x)$ is a proper function on $G$ for some (and hence any) vertex $x$.

5.5. Theorem. If $\mathrm{G}$ is a $\mathrm{CAT}(0)$-cubical group, then $\mathrm{G}$ is weakly amenable and has CowlingHaagerup constant 1.

\section{Appendix A. A Bound on the InTERsections of InTERVAls With BALLS}

We shall give a new proof, which may be of independent interest, of the following result of Chatterji and Ruane [CR05].

A.1. Theorem. Let $\mathrm{X}$ be a $\mathrm{CAT}(0)$ cube complex and let $\mathrm{x}, \mathrm{y}$ be vertices of $\mathrm{X}$. For every $\mathrm{r} \in \mathbb{N}$ the cardinality of the set $\mathfrak{C}(x, y) \cap \mathrm{B}(x, r)$ is bounded by $(r+1)^{\mathrm{d}}$, where $\mathrm{d}$ is the dimension of $\mathrm{X}$.

The proof relies on the following proposition. 
A.2. Proposition. Let $\mathrm{X}$ be a $\mathrm{CAT}(0)$ cube complex and let $\mathrm{x}$ and $\mathrm{y}$ be vertices of $\mathrm{X}$. Let $\mathrm{H} \in$ $\mathfrak{H}(x, y)$ and suppose that $x$ is adjacent to $H$. There exists a second vertex $v$ adjacent to $\mathrm{H}$ and on the same side of $\mathrm{H}$ as $\mathrm{x}$ for which

$$
\left.\mathfrak{C}(x, y)=\mathfrak{C}(x, v) \cup \mathfrak{C}\left(x^{\mathrm{op}}, \mathrm{y}\right) \quad \text { (disjoint union }\right)
$$

A.3. Remark. In fact we only require the forward inclusion for Theorem A.1.

We shall also use the fact that a hyperplane $\mathrm{H}$ in a $\mathrm{CAT}(0)$ cube complex may be given a natural $\mathrm{CAT}(0)$ cube complex structure in its own right. The set underlying $\mathrm{H}$ can be taken to be the set of vertices in $\partial \mathrm{H}_{+}$, for any fixed orientation of $\mathrm{H}$. The cubes in $\mathrm{H}$ are exactly the subsets of $\mathrm{H}$ that are cubes in X. Distance and convex hulls may be computed in $\mathrm{H}$ or in the ambient complex $\mathrm{X}$. See [Sag95].

Proof of Theorem A.1 assuming Proposition A.2 The proof is by double induction on the dimension $d$ of $X$ and the radius $r$. The base of the induction comprises two cases. The result is obvious in the case of arbitrary $d$ and $r=0$ since $B(x, 0)$ contains only $x$. It is also obvious in the case of arbitrary $r$ and $d=0$.

For the induction step, given $\mathbf{d}>0$ and $\mathbf{r}>0$, assume the result for all complexes of dimension less than $\mathbf{d}$, no matter what the value of $r$, and for all complexes $X$ of dimension $d=\mathbf{d}$ and all balls in $X$ of radius $r<\mathbf{r}$. Now assume that $\operatorname{dim}(X)=\mathbf{d}$ and let $r=\mathbf{r}$. Let $x, y \in X$ and let $H$ be a hyperplane adjacent to $x$ that separates $x$ from $y$. It follows from the proposition that

$$
\mathfrak{C}(x, y) \cap B(x, r) \subseteq\left(\mathfrak{C}\left(x^{\text {op }}, y\right) \cap B\left(x^{\text {op }}, r-1\right)\right) \cup(\mathfrak{C}(x, v) \cap B(x, r))
$$

(note that if $u \in \mathfrak{C}\left(x^{\text {op }}, y\right)$ then $d\left(x^{\text {op }}, u\right)=d(x, u)-1$ since $H$ separates $x$ from $u$ but not $x^{\text {op }}$ from $u)$. The induction hypothesis implies that

$$
\#\left(\mathfrak{C}\left(x^{\text {op }}, y\right) \cap B\left(x^{\text {op }}, r-1\right)\right) \leq r^{\mathrm{d}}
$$

Since $\mathfrak{C}(x, v) \subseteq H$ and since the dimension of $H$ is less than $\mathbf{d}$, the induction hypothesis also implies that

$$
\#(\mathfrak{C}(x, v) \cap B(x, r)) \leq(r+1)^{d-1} .
$$

Combining these estimates, we conclude

$$
\#(\mathfrak{C}(x, y) \cap B(x, r)) \leq r^{d}+(r+1)^{d-1} \leq(r+1)^{d}
$$

as required.

The remainder of the appendix is devoted to proving Proposition A.2. Let $x$ and $y$ be vertices of $X$ and let $H \in \mathfrak{H}(x, y)$ be such that $x \in \partial H$. Orient $H$ by the requirement that $x \in H_{+}$. Let $v$ be an element of the finite set $\mathfrak{C}(x, y) \cap \mathrm{H}_{+}$of maximal distance from $x$ (or equivalently of minimal distance to $y$ ). We shall show that $v$ has the properties stated in the proposition.

A.4. Lemma. Every vertex in $\mathfrak{C}(x, y) \cap \mathrm{H}_{+}$is adjacent to $\mathrm{H}$. In particular, $v$ is adjacent to $\mathrm{H}$. 
Proof. Let $u \in \mathfrak{C}(x, y) \cap \mathrm{H}_{+}$and suppose for the sake of a contradiction that $u$ is not adjacent to $\mathrm{H}$. Let $u_{0}, \ldots, u_{n}$ be a geodesic from $x$ to $y$ containing $u$. There is a first vertex $u_{j}$ on this geodesic that is not adjacent to $H$. Then of course $u_{j-1}$ is adjacent to $H$. Let $K$ be the hyperplane separating $u_{j-1}$ from $u_{j}$. Both $H$ and $K$ separate $u_{j-1}$ from $y$. It follows that there is a square containing $u_{j-1}$ as a vertex in which $\mathrm{H}$ and $\mathrm{K}$ intersect, and as in the proof of Proposition 4.9, it follows that $u_{j}$ is adjacent to $\mathrm{H}$. Contradiction.

A.5. Lemma. If $\mathrm{K} \in \mathfrak{H}(v, y)$ and if $\mathrm{K}$ is adjacent to $v$, then $\mathrm{K}=\mathrm{H}$.

Proof. If $\mathrm{K} \neq \mathrm{H}$, then since both $\mathrm{H}$ and $\mathrm{K}$ separate $v$ from $\mathrm{y}$, and since both are adjacent to $v$, the two hyperplanes intersect in a square containing $v$ as a vertex. The vertex $v^{\text {op }}$ adjacent to $v$ across $\mathrm{K}$ is therefore adjacent to $\mathrm{H}$. It is also in the interval from $x$ to $y$ and further away (by one) from $x$ than $v$. This contradicts the definition of $v$.

In the next lemma we require a small amount of the theory of normal cube paths. See [NR98a] for further information, especially the remark following Proposition 3.3.

\section{A.6. Lemma. No hyperplane $\mathrm{K} \neq \mathrm{H}$ separating $v$ and $y$ can intersect $\mathrm{H}$.}

Proof. Assume for the sake of a contradiction that such a hyperplane $\mathrm{K}$ exists. There is then a hyperplane $\mathrm{K}$ other than $\mathrm{H}$ that separates $v$ from $\mathrm{y}$ and which has the property that if $\mathrm{K}$ intersects the normal cube $\mathrm{C}$, then no hyperplane (other than $\mathrm{H}$ itself) intersecting a normal cube prior to $\mathrm{C}$ on the normal cube path from $v$ to $y$ intersects $\mathrm{H}$. Observe that the cube $\mathrm{C}$ cannot be the first cube in the normal cube path from $v$ to $y$ since by the previous lemma $\mathrm{K}$ cannot be adjacent to $v$. Let $K_{1}, \ldots, K_{d}$ be the hyperplanes spanning the normal cube $C^{\prime}$ immediately preceeding $C$. A simple separation argument based on Lemma 2.10 shows that $K \cap K_{i}$ is nonempty for each $i$. Thus, the hyperplanes $\mathrm{K}, \mathrm{K}_{1}, \ldots, \mathrm{K}_{\mathrm{d}}$ intersect pairwise, and each is adjacent to the (unique) vertex $w$ in $C^{\prime} \cap C$. By Proposition 2.7 each pair intersects in a square having $w$ as a vertex. But, the link of $w$ is a flag complex (see [BH99, Thm. II.5.20]), so that these hyperplanes intersect in a cube having $w$ as a vertex. This contradicts the definition of normal cube path according to which $\operatorname{St}\left(C^{\prime}\right) \cap C=\{w\}$.

Proof of Proposition A.2 We shall show that

$$
\mathfrak{C}(x, y) \cap \mathrm{H}_{-}=\mathfrak{C}\left(x^{\text {op }}, y\right)
$$

and

$$
\mathfrak{C}(x, y) \cap \mathrm{H}_{+}=\mathfrak{C}(x, v)
$$

Let $u \in \mathfrak{C}(x, y) \cap H_{-}$. Then $d(x, u)+d(u, y)=d(x, y)$. Since $u \in H_{-}$it follows that $\mathrm{d}\left(\mathrm{x}^{\mathrm{op}}, \mathrm{u}\right)=\mathrm{d}(\mathrm{x}, \mathrm{u})-1$. Therefore

$$
d\left(x^{o p}, u\right)+d(u, y)=d(x, u)-1+d(u, y)=d(x, y)-1=d\left(x^{o p}, y\right)
$$

so that $u \in \mathfrak{C}\left(x^{\text {op }}, y\right)$. The other inclusion in (A.1) is obvious.

It follows immediately from [Sag95, Thm. 4.13] that $\mathfrak{C}(x, v) \subseteq \mathrm{H}_{+}$. Using Corollary 2.2, if $w \in \mathfrak{C}(x, v)$ then $\mathfrak{H}(x, w) \subseteq \mathfrak{H}(x, v) \subseteq \mathfrak{H}(x, y)$ so that $w \in \mathfrak{C}(x, y)$ 
Finally, let $w \in \mathfrak{C}(x, y) \cap \mathrm{H}_{+}$. Then by Corollary 2.2 ,

$$
\mathfrak{H}(x, w) \subseteq \mathfrak{H}(x, y)=\mathfrak{H}(x, v) \cup \mathfrak{H}(v, y) \quad \text { (disjoint union) }
$$

We want to show that $\mathfrak{H}(x, w) \subseteq \mathfrak{H}(x, v)$, or equivalently that $\mathfrak{H}(x, w) \cap \mathfrak{H}(v, y)$ is empty. This is a separation argument. Indeed, suppose $K \in \mathfrak{H}(v, y)$. If $K=H$ then $K \notin \mathfrak{H}(x, w)$ by assumption. If $\mathrm{K} \neq \mathrm{H}$ then $\mathrm{K}$ and $\mathrm{H}$ are parallel by Lemma A.6. Further, $\mathrm{K}$ is contained entirely in $\mathrm{H}_{-}$, since $\mathrm{K} \in \mathfrak{H}\left(v^{\mathrm{op}}, \mathrm{y}\right)$ and both $v^{\text {op }}$ and $\mathrm{y} \in \mathrm{H}_{-}$. Now, a geodesic path from $x$ to $w$ is completely contained in $\mathrm{H}_{+}$, so cannot cross $\mathrm{H}$, and therefore cannot cross $\mathrm{K}$. That is, $\mathrm{K} \notin \mathfrak{H}(x, w)$.

\section{REFERENCES}

[BH99] M. R. Bridson and A. Haefliger, Metric spaces of non-positive curvature, Grundlehren der Mathematischen Wissenschaften [Fundamental Principles of Mathematical Sciences], vol. 319, Springer-Verlag, Berlin, 1999. MR MR1744486 (2000k:53038)

$\left[\mathrm{CCJ}^{+} 01\right]$ P.-A. Cherix, M. Cowling, P. Jolissaint, P. Julg, and A. Valette, Groups with the Haagerup property, Progress in Mathematics, vol. 197, Birkhäuser Verlag, Basel, 2001, Gromov's a-T-menability. MR MR1852148 (2002h:22007)

[CH89] M. Cowling and U. Haagerup, Completely bounded multipliers of the Fourier algebra of a simple Lie group of real rank one, Invent. Math. 96 (1989), no. 3, 507-549. MR MR996553 (90h:22008)

[CMV04] P.-A. Cherix, F. Martin, and A. Valette, Spaces with measured walls, the Haagerup property and property (T), Ergodic Theory Dynam. Systems 24 (2004), no. 6, 1895-1908.

[CN05] I. Chatterji and G. Niblo, From wall spaces to CAT(0) cube complexes, Internat. J. Algebra Comput. 15 (2005), no. 5-6, 875-885.

[Cow83] M. Cowling, Harmonic analysis on some nilpotent Lie groups (with application to the representation theory of some semisimple Lie groups), Topics in modern harmonic analysis, Vol. I, II (Turin/Milan, 1982), Ist. Naz. Alta Mat. Francesco Severi, Rome, 1983, pp. 81-123. MR MR748862 (85i:22012)

[CR05] I. Chatterji and K. Ruane, Some geometric groups with rapid decay, Geom. Funct. Anal. 15 (2005), no. 2, 311-339.

[dCH85] J. de Canniere and U. Haagerup, Multipliers of the Fourier algebras of some simple Lie groups and their discrete subgroups, Amer. J. Math. 107 (1985), 455-500.

[Far03] D. Farley, Proper isometric actions of Thompson's groups on Hilbert space, Int. Math. Res. Not. (2003), no. 45, 2409-2414.

[Gro87] M. Gromov, Hyperbolic groups, Essays in group theory, Math. Sci. Res. Inst. Publ., vol. 8, Springer, New York, 1987, pp. 75-263. MR MR919829 (89e:20070)

[Haa86] U. Haagerup, Group $\mathrm{C}^{*}$-algebras without the completely bounded approximation property, Unpublished manuscript, 1986.

[HK94] U. Haagerup and J. Kraus, Approximation properties for group $\mathrm{C}^{*}$-algebras and group Von Neumann algebras, Transactions of the AMS 344 (1994), 667-699.

[Jan93] T. Januszkiewicz, For right-angled Coxeter groups $z^{|g|}$ is a coefficient of a uniformly bounded representation, Proc. Amer. Math. Soc. 119 (1993), 1115-1119.

[Jan02] _ For Coxeter groups $z^{|\mathrm{g}|}$ is a coefficient of a uniformly bounded representation, Fund. Math. 174 (2002), no. 1, 79-86. MR MR1925487 (2003f:20061)

[Nic04] B. Nica, Cubulating spaces with walls, Algebr. Geom. Topol. 4 (2004), 297-309 (electronic).

[NR97] G. Niblo and L. Reeves, Groups acting on CAT(0) cube complexes, Geometry and Topology 1 (1997), 1-7.

[NR98a] , The geometry of cube complexes and the complexity of their fundamental groups, Topology $\mathbf{3 7}$ (1998), 621-633. 
[NR98b] G. Niblo and M. Roller, Groups acting on cubes and Kazhdan's property (T), Proc. Amer. Math. Soc. 126 (1998), no. 3, 693-699.

[Pim87] M. Pimsner, Cocycles on trees, J. Operator Theory 17 (1987), 121-128.

[Pis95] G. Pisier, Multipliers and lacunary sets in non-amenable groups, Amer. J. Math. 117 (1995), no. 2, 337376. MR MR1323679 (96e:46078)

[Sag95] M. Sageev, Ends of group pairs and non-positively curved cube complexes, Proc. London Math. Soc. 71 (1995), 585-617.

[Szw91] R. Szwarc, Groups acting on trees and approximation properties of the Fourier algebra, J. Funct. Anal. 95 (1991), no. 2, 320-343. MR MR1092129 (92e:43005)

[Va190a] A. Valette, Cocycles d'arbres et reprèsentations uniformèment bornèes, C. R. Acad. Sci. Paris, Série I 310 (1990), 703-708.

[Va190b] Les représentations uniformément bornées associées à un arbre réel, Bull. Soc. Math. Belg. Sér. A 42 (1990), no. 3, 747-760, Algebra, groups and geometry. MR MR1316222 (96i:22011a)

[Va193] _ Weak amenability of right-angled Coxeter groups, Proc. Amer. Math. Soc. 119 (1993), 13311334.

University of Hawai ‘ i, Mānoa, Department of Mathematics, 2565 McCarthy Mall, Honolulu, HI 96822-2273

E-mail address: erik@math.hawaii.edu

Department of Mathematics, Pennsylvania State University, University Park, PA 16802

E-mail address: higson@math.psu.edu 